\title{
Brain electrical activity during bench press weight training exercise
}

\author{
Pipat Engchuan', Karnt Wongsuphasawat' ${ }^{2}$, Phakkharawat Sittiprapaporn ${ }^{1,3}$ \\ ${ }^{1}$ Researcher, Brain Science and Engineering Innovation Research Group, School of Anti-Aging and Regenerative \\ Medicine, Mae Fah Luang University, Bangkok, Thailand, '2Lecturer, Department of Anti-Aging and Regenerative \\ Science, School of Anti-Aging and Regenerative Medicine, Mae Fah Luang University, Bangkok, Thailand, ${ }^{3}$ Assistant \\ Professor, Department of Anti-Aging and Regenerative Science, School of Anti-Aging and Regenerative Medicine, Mae \\ Fah Luang University, Bangkok, Thailand
}

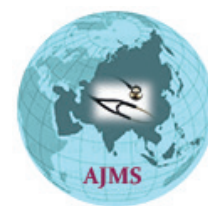

A B S T R A C T

Background: Weight training is one type of exercises which some people interest. When the body has a physical exercise with enough intensity, it can produce a positive effect on brain function by changing amplitude of electroencephalographic activity. Aims and Objective: The purpose of this investigation was to examine a more comprehensive range of the electroencephalographic activity including delta, theta, alpha, beta, and gamma brainwaves during bench press weight training exercise. Materials and Methods: Electroencephalographic activities were recorded with nine participants by using the commercial lightweight electroencephalographic device, NeuroSky Mindwave Mobile, both before and during bench press weight training exercise. Results: The present study was conducted to find out how bench press weight training exercise effected to human brainwave. The electroencephalographic activity could be recorded during bench press weight training. The findings of the present study documented increase in beta and gamma brainwaves during bench press weight training exercise. This electrophysiological changes could represent the rearrangement of different systems as a consequence of exercise. Conclusion: This bench press weight training might significantly alter the intense exercise. Future studies may want to examine how these changes in electroencephalographic activity influence or are associated with changes in cognition, affect and/or perception during/after bench press weight training exercise. The impact of peripheral physiology on electroencephalographic activity of bench press weight training exercise also needs to be examined.

Key words: Brain; Electroencephalographic activity; Bench press; Weight training
http://nepjol.info/index.php/AJMS DOI: 10.3126/ajms.v10i5.21034 E-ISSN: 2091-0576 P-ISSN: 2467-9100

\section{INTRODUCTION}

Brain is an organ that is easily affected by the physical changes, especially in the modern time when people are easily poisoned by the increasing pollution. When the poisonous substances enter the body, every organ, including the brain will be endangered. Abnormalities will occur to the brain in terms of memory, eyesight, intellect, and balance. ${ }^{1-3}$ Because the roots of all our thoughts, emotions and behaviors are the communications between neurons within our brain, our brain is made up of billions of cells called neurons which use electricity to communicate with each other. The combinations of electrical activity of the brain commonly known as brain wave pattern, because of their cyclic, 'wavelike' nature. ${ }^{4}$ These waves are produced in the brain emitting tiny electrochemical impulses of varied frequencies. These frequencies can be registered by an electroencephalogram (EEG) machine. EEG is frequently used to investigate normal and pathological conditions in brain cortex. ${ }^{4,5}$ Brain activity has been proposed to be important in examining affective and perceptual responses to acute bouts of exercise. ${ }^{6,7}$ For example, changes in brain activity may occur secondary to the metabolic changes associated with "central fatigue" during prolonged exercise. ${ }^{8-10}$ Furthermore, activity in the frontal regions of the brain has been related to affective ${ }^{7}$ and perceptual ${ }^{6}$ responses to acute bouts of exercise.

\section{Address for Correspondence:}

Dr. Phakkharawat Sittiprapaporn, Assistant Professor, Brain Science and Engineering Innovation Research Group, School of Anti-Aging and Regenerative Medicine, Mae Fah Luang University, Bangkok 10110, Thailand. Tel. No: +662 6644361, Fax No: +662 6644362. E-mail: wichian.sit@mfu.ac.th; Dr. Karnt Wongsuphasawat, Department of Anti-Aging and Regenerative Science, School of Anti-Aging and Regenerative Medicine, Mae Fah Luang University, Bangkok, Thailand, E-mail: karnt.won@mfu.ac.th (C) Copyright AJMS 
EEG is traditionally used to measure the brainwaves. Each type of brainwave is associated with one's state of consciousness and different mood state. ${ }^{11-13}$ From all types of wave, beta brainwave is seen in normal activities as well as stressful conditions or difficulties in mental concentration. On the other hand, alpha wave is seen in wakefulness, relaxed, effortless and alertness condition. Therefore, persons with high alpha wave and low in beta wave could indicate status of relaxation, arousal, less stress and better concentration. ${ }^{8}$ EEG currently is the most pragmatic way to monitor changes in brain activity in humans while exercising. Although several studies have described EEG changes immediately after exercise, very few studies have examined EEG during exercise.

Exercise is now a day well recognized as an unconventional modality for preventing or even improving brain function decline. The relationship between physical activity and brain functions has been widely investigated. Studies revealed that physical activity has been related to changes in brain functioning and the affective state of an individual. Many experiments and literature reviews concerning the effects of exercise on brain electrocortical activity was based on normal population. Previous research has shown that both acute and chronic physical exercises can induce positive effects on brain function. In addition, this is associated with improvements in cognitive performance. ${ }^{14,15}$ However, the neurophysiological mechanisms underlying the beneficial effects of exercise on cognitive processing are not well understood. ${ }^{14-16}$ Several previous studies had been proposed brain activity to be important in examining affective and perceptual responses to acute bouts of exercise including changes in brain activity that might occur secondary to the metabolic changes associated with central fatigue during prolonged exercise. ${ }^{6,7}$

Even if the relationship between brain activity and affective and perceptual responses to exercise can be understood, normal responses in EEG activity to various types of exercise must be determined. EEG currently is the most pragmatic way to monitor changes in EEG activity in humanswhile exercising. Although several studies have described EEG changes immediately after exercise, very few studies have examined EEG during exercise. Furthermore, the few studies that have examined EEG changes during exercise have typically focused on a limited range of exercise intensities and examined only a few electrode sites. Previous studies in this field focused on either a change in spectral power ${ }^{17}$ and/or event-related potentials (ERPs). ${ }^{18}$ However, to the best of our knowledge, there are no studies examining the relationship between bench press weight training, cognition, and the EEG activity.

The purpose of this investigation then was to examine a more comprehensive range of the electroencephalographic activity including delta, theta, alpha, beta, and gamma brainwaves during bench press weight training exercise. It was hypothesized that electroencephalographic activity would increases workload increases. The measurement of electroencephalographic activity of bench press weight training was mostly done by letting the participants practiced the bench press weight training and then measure brainwaves by using the effectiveness of commercially available lightweight electroencephalographic devices, named NeuroSky's Mindwave Mobile.

\section{MATERIALS AND METHODS}

\section{Participants}

The experiment was done with nine participants, aged between 22-27 years old, who were in good health, no congenital illness, no record of brain surgery, not taking medicines or drugs that affect to nervous system. Exclusion criteria were left handedness; history of medical and neurologic diseases; psychiatric disorders; head trauma; assumption of Central Nervous System active drugs in the two weeks prior to study entry; and presence of EEG abnormalities at the baseline recording. After receiving information about the aims of the study, all participants provided written consent to participate in the study that was performed according to the Helsinki declaration standards and was approved by the local institutional review board of Mae Fah Luang University, Thailand.

\section{Experimental procedure}

Recording personal information, namely, age, gender, and history of illness were recorded. Electroencephalographic recordings were taken at two different times: pre and during bench press weight training exercise. The participants arrived at the laboratory at around $10 \mathrm{a} . \mathrm{m}$. and were given 10 minutes to relax before the beginning of the first recording session. In a sound isolated room, individuals sat comfortably in a chair in order to reduce muscular tension. In baseline or pre-bench press weight training exercise, the electroencephalographic was recorded for five minutes. To minimized influences of other stimuli interferences, all other external stimuli such as lights, movements of other people and sound were restricted during the data recording. After the five minutes of resting electroencephalographic recording, participants were immediately started the bench press weight training exercising up to exhaustion. Then, participants were again placed on the chair for bench press weight training exercise and similar electroencephalographic recording session took place.

\section{Electroencephalographic recording}

Electrophysiological recording through electroencephalogram was applied in this study. The neuroheadset was used to display the output, analyze and record EEG activity. The principles of analytical check were based on the 1020 system or International 10-20 system by displaying the 
EEG output. EEG frequency analysis was performed by means of a Fast Fourier Transform algorithm, with a 2-second interval on the EEG signal. The following frequency bands were considered: delta $(0.1-3 \mathrm{~Hz})$; theta $(4-7 \mathrm{~Hz})$; alpha (8-12 Hz); beta $(13-30 \mathrm{~Hz})$; and gamma (30.5-60 $\mathrm{Hz}$ ), respectively. Data were collected with eyes closed, to observe electrical activity without external stimuli, thus minimizing possible visual artifacts.

\section{Tools and equipments}

In this study, the effectiveness of commercially available lightweight EEG devices, NeuroSky's Mindwave mobile, was used in recording the electroencephalographic activity. Via the application of a single electrode and signalprocessing unit in a headband arrangement, the NeuroSky's Mindwave mobile provided two 100-state outputs operating at $1 \mathrm{~Hz}$. Although the NeuroSky's Mindwave mobile provided a much coarser picture of brain activity than multi-electrodes or other technologies, the principle advantage of the NeuroSky's Mindwave mobile was its unobtrusive nature, which minimizes the aforementioned difficulties in conducting accurate user studies.

Traditionally, the bench press is known as an upper body strength training exercise. It consists of pressing a weight upwards from a supine position. ${ }^{19,20}$ The exercise works the pectoralis major as well as supporting chest, arm, and shoulder muscles. Historically, the bench press has evolved over the years, from floor, bridge, and belly toss variations to the methods used by bodybuilders and power lifters today. The barbell bench press is one of three lifts in the sport of powerlifting. It is used extensively in weight training, bodybuilding, and other types of training to develop the chest muscles. A barbell is generally used to hold the weight, but a pair of dumbbells can also be used. ${ }^{19-22}$ Therefore, in this study, the measuring electroencephalographic activity of bench press weight training were examined by comparing between baseline and bench press weight training exercise.

\section{Statistical analysis}

Analyze the basic demographic data of participants by using descriptive analysis with qualitative data summarized in term of mean and standard deviation. Using inferential statistic compare each type of electroencephalographic activity both baseline and during bench press weight training exercise by compared $t$-test. Every Tests were set to the statistic significant at $p<0.05$.

\section{RESULTS}

The average age of all participants was $23.56 \pm 1.59$ years old. The average of height was $177.70 \pm 5.75$, while the average of weight was $73.44 \pm 7.02$ (Table 1).
According to Table 2, it was found that delta brainwave decreased while theta and alpha brainwaves increased. However, changes of delta, theta and alpha brainwaves were not statistical significant at the 0.05 level. On the other hand, it was found that both beta and gamma brainwaves were gradually increased during bench press weight training exercise compared to the baseline with statistically significant at the 0.05 level (Table 2 and Figure 1).

When considering each brainwave, it was found that delta brainwave decreased with no statistically significant during bench press weight training exercise compared to baseline (baseline: $0.464 \pm 0.056 \mu \mathrm{V}$; bench press weight training: $0.397 \pm 0.316 \mu \mathrm{V} ; \mathrm{t}(17) ; p=0.16)$. On the other hand, theta brainwave increased with no statistical significant during bench press weight training exercise compared to baseline (baseline: $0.138 \pm 0.063 \mu \mathrm{V}$; bench press weight training: $0.202 \pm 0.954 \mu \mathrm{V}$; $\mathrm{t}(17)=1.79 ; p=0.58$ ). Similar to theta brainwave, it was found that alpha brainwave increased with no statistically significant during bench press weight training exercise compared to baseline (baseline: $0.105 \pm 0.595 \mu \mathrm{V}$; bench press weight training: $0.233 \pm 0.588 \mu \mathrm{V} ; \mathrm{t}(17)=1.66 ; p=0.16)$.

In fast waves, on the other hand, it was found that beta brainwave increased highly during bench press weight training exercise compared to baseline with statistically significant at 0.05 level (baseline: $0.357 \pm 0.194 \mu \mathrm{V}$; bench press weight training: $1.433 \pm 0.154 \mu \mathrm{V} ; \mathrm{t}(17)=0.52$; $p=0.035$ ). Finally, gamma brainwave also increased with statistically significant during bench press weight training exercise compared to baseline (baseline: $0.125 \pm 0.039 \mu \mathrm{V}$; bench press weight training: $1.134 \pm 0.067 \mu \mathrm{V} ; \mathrm{t}(17)=1.28$; $p=0.013$ )(Figure 2).

\section{DISCUSSION}

The present study is the first investigation to document intensity related increases in electroencephalographic activity following bench press weight training exercise. However, a few studies have examined changes in electroencephalographic activity as a result of differing exercise intensities but none of these studies examined electroencephalographic activity following bench press weight training. ${ }^{23,24}$ There was one study examined electroencephalographic activity during a rest period following running five or six discontinuous stages with

\begin{tabular}{|c|c|c|c|}
\hline Data & Means \pm SD & Min. & Max. \\
\hline Age & $23.56 \pm 1.59$ & 22 & 27 \\
\hline Height & $177.70 \pm 5.75$ & 169 & 188 \\
\hline Weight & $73.44 \pm 7.02$ & 65 & 86 \\
\hline
\end{tabular}


Table 2: Amplitudes of each brainwaves comparing between baseline and bench press weight training

\begin{tabular}{|c|c|c|c|c|c|}
\hline \multirow[t]{2}{*}{ Frequency bands } & \multicolumn{2}{|c|}{ Baseline } & \multicolumn{2}{|c|}{ Bench press weight training } & \multirow[t]{2}{*}{$p$-value } \\
\hline & Mean & SD & Mean & SD & \\
\hline Delta wave & 0.464 & 0.056 & 0.397 & 0.316 & 0.16 \\
\hline Theta wave & 0.138 & 0.063 & 0.202 & 0.954 & 0.58 \\
\hline Alpha wave & 0.105 & 0.595 & 0.233 & 0.588 & 0.16 \\
\hline Beta wave & 0.357 & 0.194 & 1.433 & 0.154 & $0.035^{*}$ \\
\hline Gamma wave & 0.125 & 0.039 & 1.134 & 0.067 & $0.013^{*}$ \\
\hline
\end{tabular}

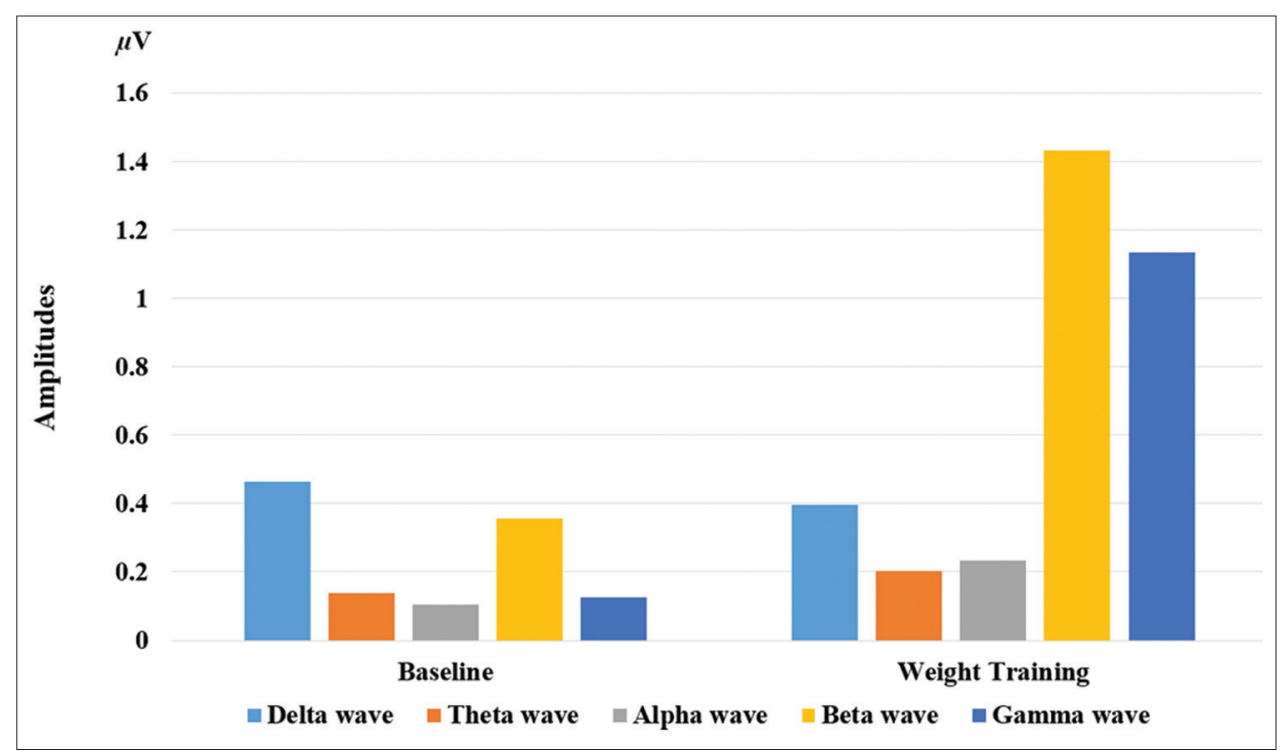

Figure 1: Amplitudes of each brainwaves comparing between baseline and bench press weight training

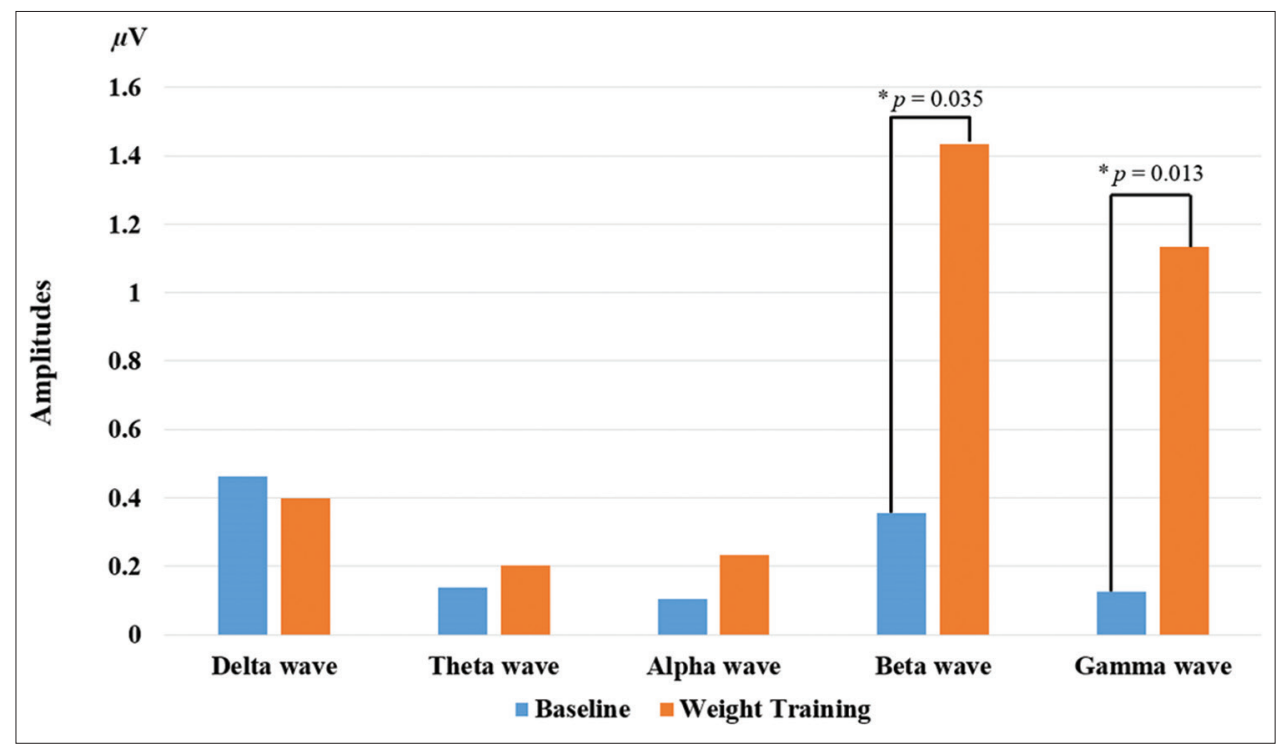

Figure 2: Comparison between baseline and bench press weight training of each brainwaves

increasing velocity. ${ }^{24}$ Therefore, the results from the present study are consistent with research from previous studies that following exercise there was an increase in electroencephalographic activity in the theta, alpha and/ or beta frequencies. ${ }^{6,24-26}$
One previous study reported decreases in alpha activity during exercise, while the present investigation observed increases in alpha activity. ${ }^{27}$ Furthermore, a previous study conducted a meta-analysis examining EEG responses during and after exercise and concluded that when 
compared to pre-exercise, alpha activity showed greater both during and following exercise. They also found that delta, theta and beta activity increased during and following exercise..$^{25}$ Similar to the present study, Crabbe and Dishman ${ }^{12}$ pointed that this previous study examined narrow ranges of the EEG frequency spectrum and only a few electrodes were monitored. We are agree with Crabbe and Dishman ${ }^{12}$ mentioned that in order for EEG to become a useful tool in understanding the impact of various experimental manipulations (e.g. distraction, fatigue, nutritional supplementation) on brain activity during exercise, the EEG responses to exercise under normal controlled conditions must be better understood.

However, the results from the present study are consistent with some previous studies showing that during and following exercise there was an increase in electroencephalographic activity in the theta, alpha and/or beta waves. ${ }^{6,12,26,28}$ The increase in the beta activity observed in the present study was similar with the results of previous studies and suggested that the augmentation in beta wave after exercise is related to greater cortical activation ${ }^{12,29-33}$ and the increase in brain regional blood flow. ${ }^{12,34}$ Similar to previous study showing that an increase in the amplitude of the beta frequency range of EEG was found during cycling at $90 \mathrm{~W}$, but found no changes at lower workloads $(50-80 \mathrm{~W}) .{ }^{35} \mathrm{In}$ addition, beta activity after exercise could be related to an attentional demand and a higher arousal level. ${ }^{30}$ However, Lardon and Polich ${ }^{36}$ reported that the observed EEG changes might be possibly seen as a consequence of exercise.

The other explanation showed that the generalized attentional or arousal effects of exercise might influence electrocortical activity in several frequencies because brain cortical systems are altered generally in response to the increased metabolic arousal that uniquely accompanies physical activity. ${ }^{37}$ In addition, hypothalamic modulation of increased metabolism and temperature during exercise are also thought to influence electrocortical activity; especially in the beta range. ${ }^{30}$ In turn, the increased cortical activation due to exercise probably influence information processing and psychological variables like attention and arousal. ${ }^{23,37}$ Thus, Kubitz and Mott ${ }^{27}$ summarized that and observed increasement in beta power at brain regions could be directly related to an excited or urgent/emergency state of mind and increased cortical activation.

Some previous studies examined electroencephalographic activity changes as a result of different environments. For instance, Nybo and Nielsen ${ }^{6}$ described changes in EEG during 1 hour of exercise at 60\% VO2max in normal $\left(18^{\circ} \mathrm{C}\right)$ and hot $\left(40^{\circ} \mathrm{C}\right)$ environments. The result showed that there was no changes in EEG as represented by percentage change from rest in the normal environment $\left(18^{\circ} \mathrm{C}\right)$. However, when exercising in the hot environment the increases in the ratio of alpha to beta frequency were found ${ }^{6}$. Finally, in order to create a better understanding of the effects of weight training exercise on brain activity, future investigations should determine if electroencephalographic activity changes as a result of weight training exercise duration alone separately from the exercise intensity. Furthermore, the presumption that changes in electroencephalographic activity during weight training exercise represents changes in cognitive brain activity and perception might not be appropriate as in order to address this issue, functional measures of brain activity (i.e., cognitive function), affect and perception must be simultaneously measured.

\section{CONCLUSION}

The present study was conducted to find out bench press weight training exercise effect on brain wave. The electroencephalographic activity could be recorded during bench press weight training. The findings of the study documented increase in beta and gamma brainwaves during bench press weight training exercise. This electrophysiological change could represent the rearrangement of different systems as a consequence of exercise. Future studies may want to examine how these changes in electroencephalographic activity influence or are associated with changes in cognition, affect and/or perception during bench press weight training exercise. The impact of peripheral physiology on electroencephalographic activity during bench press weight training exercise also needs to be examined.

\section{ACKNOWLEDGEMENT}

We thank all of the participants who involved in this study as main data provider.

\section{REFERENCES}

1. Bailey SP, Hall EE, Folger A and Miller PC. Changes in EEG during graded exerciseon a recumbent cycle ergometer. J Sports Sci Med 2008; 7:505-511.

2. Gutmann B, Mierau A, Hulsdunker T, Hildebrand C, Przyklenk A, Hollmann W, et al. Effects of Physical Exercise on Individual Resting State EEG Alpha Peak Frequency. Neural Plast 2015; 717312.

3. Moraes H, Deslandes A, Silveira H, Ribeiro P, Cagy M, Piedade R, et al. The effect of acute effort on EEG in healthy young and elderly subjects. Eur J Appl Physiol 2011; 111(1):67-75.

4. Hoque A and Mondal S. Acute aerobic exercise effects on brain wave pattern of player and non- player: A pilot study. Inter J Physical Education Sports and Health 2015; 1(4):54-56. 
5. Moreas H, Ferreire C, Deslandes A, Cagy M, Pompen F and Ribeiro P. Beta and alpha electroencephalographic activity changes after acute exercise. Arquivos de Neuropsiquoatria 2007; 65:1-8.

6. Nybo $L$ and Nielsen B. Perceived exertion is associated with an altered brain activity during exercise with progressive hyperthermia. J Appl Physiol 2001; 91:2017-2023.

7. Petruzzello SJ, Ekkekakis $P$ and Hall EE. Physical activity and affect: EEG studies. In: Psychobiology of exercise and sport. Eds: Acevedo EO and Ekkekakis P, Human Kinetics, Champaign, IL. pp. 111-128, 2006.

8. Dalsgaard MK and Secher NH. The brain at work: a cerebral metabolic manifestation of central fatigue? J Neurosci Res 2007; 85:3334-3339.

9. Davis JM and Bailey SP. Possible mechanisms of central nervous system fatigue during exercise. Med Sci Sport and Exer 1997; 29(1):45-57.

10. Newsholme EA and Blomstrand E.Tryptophan, 5-hydroxytryptamine and a possible explanation for central fatigue. Adv Exp Med Biol 1995; 384:315-320.

11. Allen JJB, Coan JA and Nazarian M. Issues and assumptions on the road from raw signals to metrics of frontal EEG asymmetry in emotion. Biol Psychol 2004; 67:183-218.

12. Crabbe JB and Dishman RK. Brain electrocortical activity during and after exercise: A quantitive synthesis. Psychophysiol 2004; 41:563-574.

13. Kakizaki T. Effects of bicycle exercise on occipital EEG amplitude in male students. Industrial Health 1988; 26:191-195.

14. Kraaier V, Van Huffelen $A C$ and Wieneke GH. Changes in quantitative EEG and blood flow velocity due to standardized hyperventilation; a model of transient ischaemia in young human subjects. Electroencephal Clin Neurophysiol 1988; 70:377-387.

15. Kraaier V, van Huffelen AC, Wieneke GH, Van der Worp HV and Bär PR. Quantitative EEG changes due to cerebral vasoconstriction. Indomethacin versus hyperventilationinduced reduction in cerebral blood flow in normal subjects. Electroencephal. Clin. Neurophysiol 1992; 82: 208-212.

16. Kubitz KA and Mott AA. EEG power spectral densities during and after cycle ergometer exercise. Res Quart Exer Sport 1996; 67(1):91-96.

17. Fumoto M, Oshima T, Kamiya K, Kikuchi H, Seki Y, Nakatani $Y$, et al. Ventral prefrontal cortex and serotonergic system activation during pedaling exercise induces negative mood improvement and increased alpha band in EEG. Behav. Brain Res 2010; 213(1):1-9.

18. Hillman $\mathrm{CH}$, Snook EM and Jerome GJ. Acute cardiovascular exercise and executive control function. Int J Psychophysiol 2003; 48(3):307-314.

19. Graham JF. "Dumbbell bench press". Strength and Conditioning,
Retrieved 7 September 2014.

20. Contreras, Bret (2011-12-15). "The Best Damn Bench Press Article Period". T Nation. Retrieved 14 August 2014.

21. "Men's Raw World Records (Open)". PowerLiftingWatch.com. Retrieved 01 November 2015.

22. "Barbell Bench Press Video Exercise Guide \& Tips - Muscle \& Strength". Muscleandstrength.com. Retrieved 14 August 2014.

23. Kamijo K, Nishihira Y, Hatta A, Kaneda T, Kida T, Higashiura T, et al. Changes in arousal level by differential exercise intensity. Clin Neurophysiol 2004; 115:2693-2698.

24. Mechau D, Mucke S, Weiss $M$ and Liesen $H$. Effect of increasing running velocity on electroencephalogram in a field test. Eur $\mathrm{J}$ Appl Physiol 1998; 78(4):340-345.

25. Crabbe JB and Dishman RK. Brain electrocortical activity during and after exercise: A quantitive synthesis. Psychophysiol 2004; 41:563-574.

26. Nielsen B, Hgldig T, Bidstrup F, Gonzalez-Alonso $\mathrm{J}$ and Christoffersen GRJ. Brain activity and fatigue during prolonged exercise in the heat. Eur JAppl Physiol 2001; 442:41-48.

27. Kubitz KA and Mott AA. EEG power spectral densities during and after cycle ergometer exercise. Res Quart Exer Sport 1996; 67(1):91-96.

28. Kamijo K, Nishihira Y, Hatta A, Kaneda T, Kida T, Higashiura T, et al. Changes in arousal level by differential exercise intensity. Clin Neurophysiol 2004; 115:2693-2698.

29. Youngstedt S, Dishman R, Cureton K and Peacock L. Does body temperature mediate anxiolytic effects of acute exercise? J Appl Physiol 1993; 74:825-831.

30. Nielson B and Nybo L. Cerebral changes during exercise in the heat. Sports Med 2003; 33:1-11.

31. Bailey S, Hall E, Cain J, Miller P and Folger S. Changes in the brain activity during a graded exercise test on a recombert cycle ergometer (Abstr.). Med Sci Sports Exerc 2004; (Suppl):S286.

32. Doyle LM, Yarrow K and Brown P. Lateralization of eventrelated beta desynchronization in the EEG during precued reaction times tasks. Clin Neurophysiol 2005; 116:1879-1888.

33. Ogoh S, Fadel PJ and Zhang R. Middle cerebral artery flow velocity and pulse pressure during dynamic in humans. Am J Physiol Heart Circ Physiol 2005; 288:1526-1531.

34. Orlandi $G$ and Murri L. Transcranial Doppler assessment of cerebral flow velocity at rest and during voluntary movements in young and elderly healthy subjects. Int J Neurosci 1996; 84:45-53.

35. Kakizaki T. Effects of bicycle exercise on occipital EEG amplitude in male students. Industrial Health, 1988; 26:191-195.

36. Lardon $M$ and Polich J. EEG changes from long-term physical exercise. Biol Psychol 1996; 44:19-30.

37. Tomporowski $\mathrm{P}$ and Ellis $\mathrm{N}$. Effects of exercise on cognitive process; a review. Psycho Bull 1986; 99:338-346.

\section{Authors Contribution:}

PE-Concept and design of the study, statistically analyzed and interpreted; KW-Concept and design of the study; PS-Concept and design of the study, manuscript preparation, statistically analyzed and interpreted, critical revision of the manuscript.

\section{Work attributed to:}

Brain Science and Engineering Innovation Research Group, School of Anti-Aging and Regenerative Medicine, Mae Fah Luang University, Bangkok 10110,

Thailand.

\section{Orcid ID:}

Pipat Engchuan- (1) https://orcid.org/0000-0002-8764-2932

Dr. Karnt Wongsuphasawat- (i) https://orcid.org/0000-0001-6570-0663

Dr. Phakkharawat Sittiprapaporn- 10 https://orcid.org/0000-0002-4103-9396

Source of Support: Brain Science and Engineering Innovation Research Group, Mae Fah Luang University (MFU-Grant), Thailand, Conflict of Interest: None. 